\title{
Should we be screening for early diabetic renal disease?
}

Renal disease is an important and costly complication of diabetes, both in human and economic terms. Knowledge of the epidemiology of diabetic nephropathy has advanced considerably in recent years. ${ }^{1}$ The natural progression of disease in those who develop renal failure is fairly well understood. Early nephropathy can be identified by a simple urine test and the course of disease can be modified by both drug and non-drug intervention. So should we systematically screen the diabetic population to reduce the incidence of renal failure?

\section{The disease}

The incidence of renal failure in diabetes is not known, but in 1985 and 1986 there were 3753 death certificates from England and Wales which mentioned diabetes with renal failure. Diabetes is now the single commonest diagnosis in new dialysis patients in the USA ${ }^{2}$ and accounts for a rapidly increasing proportion of those receiving replacement therapy in most European countries. ${ }^{3}$ This should not be taken to mean that the incidence of diabetic nephropathy is rising, since acceptance of diabetic patients for renal replacement therapy undoubtedly reflects availability of treatment and medical attitudes to patient suitability. In the USA, the cost of treatment for diabetic end stage renal disease has been estimated at $\$ 1.8$ billion each year and is rising rapidly. Although renal failure occurs in both insulin dependent and non-insulin-dependent diabetes, most of the available data is about insulin dependent diabetes (IDDM) to which the following applies.

The hallmark of diabetic nephropathy is a rise in urinary protein (mainly albumin) excretion. When urinary albumin excretion is about 10 times higher than normal, it can be detected by semiquantitative strip reagents, so called "dipstick positive proteinuria". Although renal function is usually normal at this stage, proteinuria is an ominous sign. Accompanied by raised blood pressure, it heralds a linear decline in renal function and a median survival, without renal support, of only 7-10 years. The terms "clinical nephropathy" and "overt nephropathy" refer to this triad of proteinuria, hypertension, and falling glomerular filtration rate.

Studies from Denmark and the USA show that the cumulative incidence of proteinuria after $\mathbf{4 0}$ years of IDDM is around $45 \%$. The incidence rate rises rapidly after 10 to 20 years of diabetes to give a peak prevalence of $21 \%$ after $20-25$ years and then falls steeply with longer duration. This implies that only a subgroup of IDDM patients is at risk of clinical nephropathy, even though the characteristic histology of glomerular sclerosis is found in over $90 \%$ of patients after 10 years of diabetes.

Urinary albumin concentrations which are too low to be detected by dipstick tests can be measured by more sensitive immunoassays and excretion rates calculated from timed urine collections. The rate of urinary albumin excretion in healthy non-diabetics is normally less than $15 \mu \mathrm{g} / \mathrm{min}$ and dipstick positive proteinuria equates with an excretion rate of over
$200 \mu \mathrm{g} / \mathrm{min}$. Raised urinary albumin excretion between these methodologically defined limits is often referred to as "microalbuminuria", although it is clear that it is a continuous, not a categorical, variable.

It is generally believed (see below) that gradual increases in albumin excretion and blood pressure precede the development of clinical nephropathy by 10 to 14 years. Thus a susceptible patient, with IDDM since the age of 14 years, might expect to develop microalbuminuria around the age of 25 years, clinical nephropathy before 40 , and end stage renal failure before the age of 50 . Assuming this to be a reasonable description of the natural history, there should be ample opportunity to identify people at high risk of diabetic nephropathy, ie, those with microalbuminuria.

\section{The screening test}

For urinary albumin to be an appropriate screening test, it must be possible to define a rate of urinary albumin excretion which predicts clinical nephropathy. Four historical follow up studies in small numbers of patients have concluded that raised albumin excretion is a strong predictor of clinical nephropathy. ${ }^{4-7}$ In each study, the sensitivity and specificity of the test were optimised by retrospectively selecting the cutoff level which would predict a positive follow up result, namely a urinary albumin excretion rate of $>200 \mu \mathrm{g} / \mathrm{min}$ or dipstick positive proteinuria after a mean follow up of 6 to 14 years. With cutoff levels varying from $>15 \mu \mathrm{g} / \mathrm{min}$ to $>70 \mu \mathrm{g} / \mathrm{min}$, optimised sensitivity ranged from $70 \%$ to $100 \%$ and specificity from $94 \%$ to $100 \%$. If the prevalence of microalbuminuria in the population being screened was $15 \%$, the predictive value of a positive test would range from $75 \%$ to $100 \%$, and that of a negative test from $96 \%$ to $100 \%$. Given that intraindividual variation in urinary albumin excretion is high, the test characteristics calculated in these studies look promising. However, these studies did not take account of duration of diabetes and glycaemic control, which are major risk factors for diabetic nephropathy. In three of the studies, ${ }^{5-7}$ the differences in mean duration of diabetes between positively screened (14,17, and 19 years) and negatively screened patients $(9,13$, and 15 years respectively) span the period when the incidence of proteinuria rises most steeply with duration. Further questions about the predictive value of urinary albumin excretion are raised by two large cross sectional studies, which show that the prevalence of microalbuminuria continues to rise after more than 30 years of diabetes. Since the incidence of nephropathy declines markedly after 25-30 years, these findings imply that progression from microalbuminuria to proteinuria may not be the rule, at least in people with longstanding disease. Longitudinal studies which take account of confounding factors are needed to determine more precisely the risk of progression to clinical nephropathy at different levels of urinary albumin excretion.

Measurement of the rate of urinary albumin excretion requires a timed urine collection, but there is good correlation 
between overnight albumin/creatinine ratio and albumin excretion, and "microalbuminuria detecting dipsticks" are becoming available for use on "spot" urines. Such tests are inexpensive, simple to perform, acceptable to patients, and could be repeated with timed urine collections in order to improve the sensitivity and specificity of screening.

\section{The intervention}

There should be an acceptable and effective treatment for positively screened patients, and early treatment should be preferable to late treatment. Although many more diabetic patients are now accepted for renal replacement therapy than they were a few years ago, dialysis remains an unsatisfactory treatment, and the outcome of transplantation is still worse in diabetic patients compared with non-diabetics. Earlier treatment with antihypertensive drugs has aroused much interest because of the close link between diabetic nephropathy and high blood pressure. Particular attention has focused on angiotensin converting enzyme (ACE) inhibitors, owing to the controversial claim that these drugs exert a specific protective effect on renal function by reducing intraglomerular pressure. Clearly, what needs to be shown is that intervention reduces the risk of renal failure. Three studies"-10 are commonly quoted as establishing "beyond doubt" the benefit of antihypertensive treatment in clinical nephropathy. In fact all three studies are before/after comparisons of long term antihypertensive therapy in 6,10 , and 14 patients respectively, who experienced a fall in the rate of linear decline in glomerular filtration rate following intervention. Another study found no difference in the rate of decline of glomerular filtration rate between 15 patients given 12 months ACE inhibitor therapy and 15 untreated controls, although protein excretion increased by $55 \%$ in the control group and fell by $11 \%$ in the treated group. A recent study concluded that antihypertensive treatment has improved prognosis in diabetic nephropathy by comparing a 10 year survival rate of $82 \%$ in 45 proteinuric patients, 37 of whom received antihypertensive treatment between 1974 and 1978, with 10 year survival rates of $23-50 \%$ in proteinuric patients followed between 1940 and 1970 . However, it is well known that comparisons based on controls from other studies are highly subject to bias. As patients with diabetic nephropathy have high cardiovascular morbidity and mortality, there is added incentive to lower their blood pressure, although the extent to which these patients are likely to benefit from antihypertensive treatment is uncertain, because estimates of coronary risk reduction come largely from trials in nondiabetics.

Since proteinuria identifies patients who already have quite advanced disease, beset by additional diabetic complications, attention has turned to earlier stages in the natural history where the potential for disease modification might be greater. Indeed improved glycaemic control has been shown to reduce urinary albumin excretion in patients with microalbuminuria, but not proteinuria. But only short term benefit has been shown, and this needs to be considered alongside the formidable problems of maintaining excellent control and the increased risk of severe hypoglycaemia which that may bring.

Hypertension was initially thought to be a late and therefore secondary feature of diabetic nephropathy, but it is now clear that blood pressure begins to rise earlier in the natural history. There is, in fact, much debate about whether the rise in blood pressure is a cause, rather than a consequence, of increased urinary albumin excretion, but the appropriateness of screening does not rest on resolution of this argument. Trials of up to 12 months duration in small numbers of patients with microalbuminuria have shown that antihypertensive therapy can reduce urinary albumin excretion and uncontrolled studies have reported lowered values for up to five years.
Dietary protein restriction may also be beneficial in patients with impaired renal function, but studies in diabetic patients have mostly been small, uncontrolled, or unadjusted for confounding.

\section{Ethical and practical issues}

If treatment for early diabetic renal disease were considered appropriate, would a systematic screening programme work in practice? There is good reason to think so, particularly as dipstick tests capable of detecting raised urinary albumin concentrations are becoming available for use in diabetic clinics or in the home. Screening to prevent secondary complications in an already "diseased" population does not carry quite the same implications as a primary screening programme, eg, for breast cancer. However, it is difficult to know what the likely ratio of true to false positive screening results would be, and the consequences of unnecessary treatment arising from false positive results are not clear. While the optimum frequency of screening is unknown, neither cost nor acceptability to patients is likely to present a major problem.

\section{Likely costs versus benefits}

One of the crucial unanswered questions is "How safe and effective is long term intervention". Another is "What level of urinary albumin excretion would optimise the risk-benefit ratio of screening induced treatment?" These kinds of questions could be answered by a large randomised controlled trial of patients with microalbuminuria. The results of such a trial could also be used to estimate the likely economic implications of widespread screening. The choice of antihypertensive agent will be important since the cost of a year's treatment varies enormously between different drugs. At present all that can be said is that the personal costs borne by patients requiring renal support are likely to outweigh those incurred by screening and antihypertensive therapy. Further information is needed to estimate the comparative costs to the health service.

\section{Conclusions}

In order to answer the question "Should we be screening diabetic patients for early renal disease?" more information about the efficacy and safety of intervention is needed. Although much is known about the adverse effects of $\beta$ blockers and diuretics, experience with ACE inhibitors is still relatively limited. Several features of the disease and the screening test are compatible with a successful screening programme, but no trial has shown that early intervention reduces renal morbidity or mortality. While short term clinical trials do not provide enough information for decisions about screening, they undoubtedly influence clinical practice. Recent recommendations for the management of patients with microalbuminuria include drug treatment for blood pressures of $140 / 85 \mathrm{~mm} \mathrm{Hg}$, and perhaps as low as $130 / 80 \mathrm{~mm} \mathrm{Hg}$. In response to a questionnaire survey of diabetic clinics taking part in a European study of diabetic complications (EURODIAB), 19 out of 21 clinics claimed to include measurement of urinary albumin excretion as part of the routine care of insulin dependent patients. Attitudes to treatment of raised albumin excretion or mildly raised blood pressure were often uncertain, but $37 \%$ of responding clinicians felt that it would be important to give antihypertensive drugs, presumably for life, to an insulin dependent patient with microalbuminuria and a blood pressure between $130 / 80$ and $140 / 90 \mathrm{~mm} \mathrm{Hg}$. The danger is that current belief in the benefit of early treatment will damage efforts to seek more convincing evidence for this through a 
randomised controlled trial of antihypertensive therapy or other intervention. It is all too easy to end up with an enthusiastic screening programme of unproven benefit which is hard to evaluate. Screening for diabetic renal disease deserves a better fate.

J Stephenson

EURODIAB IDDM Complications Study

Department of Epidemiology and Public Health University College and Middlesex School of Medicine

University of London 66-72 Gower Street London WC1E 6EA United Kingdom

1 Selby JV, Fitzsimmons SC, Newman JM, Katz PP, Sepe S, Showstack J. The natural history and epidemiology of diabetic nephropathy. $\mathfrak{F} A M A$ 1990; 263: 1954-60.
2 Teutsch S, Newman J, Eggers $P$. The problem of diabetic renal failure in the United States: an overview. Am f Kidney Dis 1989; 13: 11-13.

3 Brunner FP, Brynger H, Challah S, et al. Renal replacement therapy in patients with diabetic nephropathy, 1980-1985. Nephrol Dial Transplant 1988; 3: 585-95.

4 Mogensen CE. Predicting diabetic nephropathy in insulin-dependent patients. N Engl f Med 1984; 311: 89-93.

5 Viberti GC, Hill RD, Jarrett RJ, Argyropoulos A, Mahmud U, Keen H. Microalbuminuria as a predictor of clinical nephropathy in insulindependent diabetes mellitus. Lancet 1982 ; i: 1430-2.

6 Mathiesen ER, Oxenboll B, Johansen K, Svendsen PA, Deckert T. Incipient nephropathy in type 1 (insulin-dependent) diabetes. Diabetologia 1984; 26: 406-10.

7 Parving H-H, Oxenboll B, Svendsen PA, et al. Early detection of patients at risk of developing diabetic nephropathy: a longitudinal study of urinary albumin excretion. Acta Endocrino

8 Parving H-H, Andersen AR, Smidt UM, Hommel E, Mathiesen ER, Svendsen PA. Effect of antihypertensive treatment on kidney function in diabetic nephropathy. BMF 1987; 294: 1443-7.

9 Mogensen GC. Long-term antihypertensive treatment inhibiting progression of diabetic nephropathy. BMF 1982; 285: 685-8.

10 Bjorck S, Mulec H, Granerus G, Herlitz H, Aurell M. Beneficial effects of angiotensin converting enzyme inhibition on renal function in patients with diabetic nephropathy. $B M \mathcal{F} 1986 ; 293$ : 471-4. 